\title{
Théologiques
}

\section{Le charisme comme énigme}

\section{Barbara Thériault et Jean Duhaime}

Volume 17, numéro 1, 2009

Les charismes

URI : https://id.erudit.org/iderudit/039495ar

DOI : https://doi.org/10.7202/039495ar

Aller au sommaire du numéro

Éditeur(s)

Faculté de théologie et de sciences des religions, Université de Montréal

ISSN

1188-7109 (imprimé)

1492-1413 (numérique)

Découvrir la revue

Citer ce document

Thériault, B. \& Duhaime, J. (2009). Le charisme comme énigme. Théologiques, 17(1), 5-14. https://doi.org/10.7202/039495ar d'utilisation que vous pouvez consulter en ligne.

https://apropos.erudit.org/fr/usagers/politique-dutilisation/ 
Théologiques 17, no 1 (2009) p. 5-14

LIMINAIRE

\section{Le charisme comme énigme}

Barbara THÉRIAULT*

Sociologie

Université de Montréal

Jean DUHAIME*

Théologie et sciences des religions

Université de Montréal

Un spectre hante ce dossier - le spectre de Max Weber. Bien que les éditeurs n'aient pas, sauf exceptions, explicitement incité les auteurs du dossier à traiter du sociologue dans leurs textes, il est omniprésent. La plupart ont, en effet, associé son nom à la notion de charisme et ont fait usage de ses travaux comme filtre pour aborder différents aspects liés à cet objetlimite des sciences humaines et sociales.

Il est vrai que Max Weber a popularisé le concept de charisme, qu'il a emprunté à la théologie de saint Paul à travers les écrits de Rudolf Sohm, juriste luthérien. Sous la plume de Paul, ce concept exprime d'abord le «don de la grâce» qui peut prendre de multiples formes, comme les auteurs du dossier le soulignent. À l'instar de concepts tels que ceux de «paria» ou

* Barbara Thériault est professeur agrégé au Département de sociologie et au Centre canadien d'études allemandes et européennes de l'Université de Montréal. Elle est rédactrice en chef de la revue Sociologie et sociétés. Elle a conjointement obtenu son Ph.D. du Centre Max-Weber de l'Université d'Erfurt et de l'Université Libre de Bruxelles. Si la sociologie wébérienne constitue le cadre général de ses recherches, ses objets d'études ont changé: des Églises en Allemagne de l'Est, elle a accordé de plus en plus d'attention aux services policiers, toujours en Allemagne.

* Jean Duhaime est professeur titulaire en interprétation biblique à la Faculté de théologie et de sciences des religions de l'Université de Montréal. Il dirige un groupe de chercheurs québécois qui participent à la publication de La Bibliothèque de Qumrân (Paris, Cerf, 2008-). Il a récemment achevé un commentaire de la Règle de la guerre de Qumrân (1QM) pour une anthologie américaine intitulée The Lost Bible. Ancient Jewish Writings Outside of Scripture.

(C) Revue Théologiques 2009. Tout droit réservé. 
d' "ascèse ", Weber a extirpé le terme "charisme» d'un contexte religieux précis pour l'utiliser dans ses écrits de sociologie politique et ses études comparées des religions, pour décrire et rendre compte de phénomènes au cœur des grands bouleversements historiques. Ainsi conçu, le charisme est un idéaltype, il est une création de l'observateur, une ligne de contour qui n'existe pas dans la réalité. Weber ne se lasse pas de répéter que l'idéaltype est un outil qui nous sert à décrire une réalité et non à la confirmer. Parce que la réalité empirique ne lui correspond que rarement, presque jamais complètement et, dans certains cas, pas du tout, le procédé agace et suscite une critique qui se manifeste dans certains textes de ce dossier.

Tout en continuant sa "carrière ", le concept de charisme élaboré par Weber pour les sciences sociales a réintégré le terrain de la théologie, non sans tribulations, et été soumis à une recontextualisation. C'est ainsi que se sont développés jusqu'à nos jours, des travaux sur les prophètes bibliques traités comme chefs charismatiques contestant les autorités politiques et dénonçant les problèmes sociaux ou religieux de leur temps, des analyses sociologiques des charismes pauliniens, des études sur les tensions entre charisme et institution dans les Églises et les communautés, ou encore sur de nouveaux mouvements religieux à caractère charismatique. Si l'on ne distingue pas toujours clairement dans ces études ce qui est du ressort de la théologie et de la sociologie, on y retrouve dans tous les cas la même insistance sur le fait que le talent particulier d'une personne doit être discerné par la communauté qui, en vertu de ce talent, reconnait son «ministère » ou l'appelle à l'exercer.

Parce qu'il lie l'ordinaire et l'extraordinaire, parce qu'il renvoie à l'unique et à l'exceptionnel, le charisme s'avère énigmatique. Sans cesse il nous échappe, comme s'il passait à travers les mailles des filets que nous tissons avec nos outils méthodologiques. C'est sans doute en partie ce caractère fuyant qui explique la grande variété de réactions qu'il suscite chez les nombreux auteurs dont il traverse les travaux: il les incite tantôt à transgresser les frontières de leur discipline, tantôt à les délimiter encore plus strictement. Il est à cet effet intéressant de constater que, parmi les collaborateurs de ce dossier, des sociologues ont pris le charisme comme point de départ d'une discussion sur les frontières établies de leur discipline, ce qui les amène soit à questionner l'entreprise sociologique (Johannes Weiss), soit à la redéfinir en orientant notre regard vers des perceptions souvent laissées dans l'ombre - comme en témoigne l'attention portée au corps dans les textes d'Isabelle Kalinowski et de Camille Joseph. Certains théologiens, de leur côté, se sont plutôt distancés de la sociologie et de la 
méthode idéaltypique, considérant par exemple les charismes pauliniens et le charisme wébérien comme de "faux-amis» (Alain Gignac), ou se réappropriant le concept de plein droit pour l'appliquer à un effort de discernement du «charisme collectif» d'une communauté religieuse internationale (André Charron).

Même si chacun des auteurs en fait un usage différent, les travaux de Weber sur le charisme jettent des ponts entre les textes du présent dossier. Qui plus est, la lecture des textes suggère qu'un idéaltype particulier du charisme sous-tend plusieurs d'entre eux. Dans quelle mesure ces contributions font-elles état ou traduisent-elles les problèmes inhérents au charisme comme objet d'étude des sciences sociales et humaines? Quelles clarifications ou quelles pistes proposent-elles, le cas échéant? Telle est la réflexion à laquelle nous sommes invités.

\section{L'idéaltype d'une lecture dominante du charisme}

Dans les études sociologiques contemporaines portant sur le charisme, on retrouve une lecture dominante qui se revendique souvent de Max Weber ou d'une approche inscrite dans son prolongement. Que ce soit pour s'en rapprocher ou s'en éloigner, c'est à cette tendance que puisent majoritairement les auteurs du dossier. Gardant à l'esprit la littérature sociologique récente sur le charisme et l'enjeu de ce dossier (le traitement d'un objetlimite), il est possible de tracer les contours, de construire l'idéaltype d'une compréhension du charisme tenue aujourd'hui pour adéquate par de nombreux chercheurs en sciences sociales, et qui pourrait, ou non — là n'est pas la question - être cautionnée par Max Weber. Elle se caractérise par un certain nombre de traits distinctifs qu'il convient ici d'accentuer.

D'abord, le charisme n'y est pas pensé comme le propre d'une personne, mais bien plutôt comme résidant au cœur d'une relation sociale dans un contexte de domination. Cette relation implique un chef charismatique (ou un groupe charismatique) et des adeptes. C'est la prétention charismatique du chef et la volonté d'obéissance des adeptes qui permettent à l'observateur d'identifier une domination charismatique: le chef (ou son équivalent) demeure charismatique aussi longtemps que ses adeptes lui confèrent un tel statut en lui témoignant obéissance, et aussi longtemps que lui-même a foi en son autorité. Pour se pérenniser, la domination charismatique doit être confirmée. Selon cette lecture idéaltypique, le charisme est soumis à de fortes tensions et c'est justement ce qui lui confère son caractère instable. De cette lecture des tensions découle une hypothèse particu- 
lière voulant que, sous le poids du quotidien, la domination charismatique tende soit à se transformer (en des formes traditionnelles et rationnelles bureaucratiques), soit à se dépersonnaliser et s'institutionnaliser (à l'exemple du charisme de fonction). Précisons enfin qu'une posture méthodologique se greffe à cette lecture: l'observateur n'a pas à déterminer la validité objective de la prétention charismatique. La reconnaissance de la légitimité d'une autorité charismatique est le fait du groupe qui accepte de s'y soumettre.

Ainsi défini et "objectivé ", le charisme est aujourd'hui avant tout étudié dans ses manifestations quotidiennes. Pour reprendre la formule de Martin Albrow, le charisme est, dans une telle perspective, "un mystère qui devient un fait dans une situation" $(1990,172)$. Cette position est, à plusieurs égards, fort convaincante. Elle nous permet de saisir, du moins partiellement, un objet qui, sinon, menace toujours de se dérober, et ainsi d'identifier ses manifestations contemporaines. Cette approche, si attentive aux tensions propres au charisme, renvoie à une autre tension, perceptible autant chez Max Weber que chez ses interprètes, entre la volonté de rendre compte et d'expliquer une réalité, et celle d'en préserver la dimension unique dont la dépouille si facilement l'analyse ${ }^{1}$. Si la lecture dominante esquissée nous laisse aussi insatisfaits, ce pourrait bien être dû au fait qu'en ramenant le charisme à une relation sociale observable en expressions concrètes, elle le désenchante par le fait même et lui soustrait ainsi son caractère magique; elle réduit le charisme à une "catégorie résiduelle " des sciences sociales, que l'on invoque seulement en dernier recours.

\section{L'idéaltype du charisme mis à l'épreuve}

Dans le langage courant, le charisme se présente toujours comme la qualité étonnante de certaines personnalités religieuses, politiques ou artistiques, qui sont alors "plus grandes que nature ». Situé à la frontière de l'extraordinaire et du quotidien, il rappelle les traits du génie, du héros ou du «geste

1. Ce dilemme est illustré par le traitement du génie par Norbert Elias dans Mozart, sociologie d'un génie. S'inspirant de Wolfgang Hildesheimer (1991 [1979]), pour qui Mozart esquive toute explication, le sociologue tente de "nous aider à mieux comprendre et à expliquer ce qui nous paraît incompréhensible dans notre existence sociale $(1991,24)$ par le recours aux figurations de l'époque et à la position du personnage dans celles-ci. S'il sent tout de suite le besoin d'ajouter: "Mon objectif n'est [...] pas de détruire, ni de réduire ce génie, mais de rendre plus compréhensible sa situation humaine» $(1991,24)$, c'est qu'Elias n'atteint que partiellement son but. 
admirable » et semble ainsi rester irrémédiablement perméable à l'objectivation. Doit-on en conséquence se résigner à l'impossibilité d'appréhender ce mystère insaisissable ou peut-on espérer résoudre l'énigme du charisme grâce à des approches séculières des sciences sociales et humaines? Ou ne vaudrait-il pas mieux reformuler la question du charisme et ainsi esquiver ce dilemme? Ce sont précisément ces questions qui sont à l'origine du présent dossier. Si dans leurs textes les auteurs empruntent le plus souvent à l'idéaltype énoncé plus haut, ils le reformulent toutefois chacun à leur façon - et, par le fait même, ils mettent en évidence les caractéristiques et les frontières de leur propre champ. Mais ces frontières, ne serait-ce pas le caractère même du charisme comme objet d'étude qui invite à les transgresser?

\subsection{Le charisme sous le regard de la sociologie et de la littérature}

Les premiers textes du dossier sont critiques de la lecture sociologique dominante du charisme. Dans «Le charisme. Aux frontières de la sociologie ", le sociologue Johannes Weiss se penche sur les écrits de Max Weber et plaide pour l'étude de l'exceptionnel, du charisme original en sociologie. C'est ce charisme authentique qu'il convient de distinguer, de "discerner » pour reprendre le verbe utilisé dans un contexte religieux, des charismes faux ou fabriqués. À l'encontre de lectures récentes (notamment celles de Breuer, de Gebhardt, de Roth et de Schluchter), il ne privilégie pas l'étude du charisme transformée, c'est-à-dire son écho dans des formes quotidiennes ou dépersonnalisées, mais bien le charisme dans son aspect le plus magique et unique. Pour Weiss, le charisme est la grande force de changements irrémédiablement liée à des individus. À cet effet, l'auteur revient sur une idée esquissée dans un texte antérieur (Weiss 2001, 86) dans lequel il se proposait de jeter les bases d'une sociologie des grands individus, dans toute leur singularité. Il ne faut cependant pas se méprendre: l'étude du caractère individuel proposée par Weiss est intimement liée au général. S'appuyant sur Kierkegaard, il en dénote le caractère double: «d'une part, "au sens de la distinction ou du talent spécial"; d'autre part, "au sens où tout homme sans exception peut et doit l'être” "(p. 16). Examinée à la lumière de la position idéaltypique développée plus haut, l'interprétation de Weiss provoque: elle en appelle en effet à une posture qui détonne d'une démarche qui se concentrerait plutôt sur l'analyse de conditions sociales à l'origine du charisme. Pour Weiss, "ces réalités ne sont pas "conditionnées socialement", mais elles peuvent influencer, voire modifier de façon radicale les conditions sociales $»$ (p. 29). 
Dans «Le visage du charisme. Une page de Proust », Isabelle Kalinowski nous présente le charisme sous un jour inconnu de la lecture dominante esquissée ci-haut. À partir de deux scènes tirées de l'œuvre romanesque de Marcel Proust, À la recherche $d u$ temps perdu, elle expose la relation entre deux registres d'adhésion: la croyance traditionnelle et la croyance charismatique. À travers le récit, le regard et la perception du jeune Marcel de la duchesse de Guermantes, nous sommes témoins des différents moments qui participent à la naissance du charisme. Isabelle Kalinowski se livre à une fine lecture qui, parce qu'elle est attentive à la perception des corps, insiste sur le jeu de reconfiguration et de passage d'un registre d'adhésion à l'autre. Ce faisant, elle renverse, précise, deux aspects du charisme tel qu'on l'entend fréquemment: la tradition produit des effets magiques; et la croyance charismatique est le produit - et non le contraire - d'une déception, d'une mise à l'épreuve de la croyance traditionnelle face à la réalité. C'est là que jaillit la croyance charismatique qui crée quelque chose de nouveau dans le regard de son porteur. Sans s'y réduire, cet effet du charisme s'apparente à ceux de l'amour ou de la religion tels qu'ils sont appréhendés par Georg Simmel et William James.

Rejoignant à la fois l'idée des grands personnages et le traitement du corps dans la littérature, Monique Moser-Verrey déploie une analyse de la représentation du "geste admirable ", un objet qu'elle associe au charisme. Rapidement, son regard se porte sur les stratégies de représentations du corps sensible dans le roman des Lumières, plus particulièrement dans les nouvelles de Friedrich Schiller. Une question est au cœur de l'article: comment le poète donne-t-il à voir aux lecteurs, par ses mots, des corps vivants et des gestes admirables? Par l'étude du sublime, du pathos, et ses déplacements dans ce corpus, "Gestes admirables ou le langage saisissant du corps dans la poétique de Friedrich Schiller » met en lumière les traits spécifiques des nouvelles allemandes de l'époque.

Les deux textes suivants sont des exemples - plus ou moins explicites - de l'utilisation d'une approche et du vocabulaire wébériens. À partir de la vie et du projet d'édition politique de François Maspero, Camille Joseph expose les tensions liées à l'entreprise d'édition politique engagée (notamment les tensions entre l'image de succès et le projet d'une entreprise qui se veut anti-capitaliste; entre un personnage charismatique et sa volonté de gestion collective; entre l'origine bourgeoise de l'éditeur et son parcours militant). En l'abordant sous un angle original, Camille Joseph ajoute une dimension supplémentaire à la lecture dominante du charisme, plus soucieuse des conduites de vie et du destin individuel. Plus que les tensions et 
l'ambivalence de l'entreprise elles-mêmes, ce sont les conséquences de ces tensions, la souffrance qu'elles génèrent chez l'individu qui les affronte, qui sont au centre de l'article. En effet, l'originalité de "Charisme et souffrance de l'éditeur politique: François Maspero» est le traitement de la souffrance, autant psychique que physique, comme preuve, confirmation du charisme de son porteur. Conséquence non voulue de l'action de Maspero, cette souffrance est également ancrée, comme l'auteur le souligne dans la deuxième partie de son texte, dans l'histoire de la famille Maspero, et fait écho au destin du grand frère militant mort au combat.

Parmi les études de ce dossier, celle de Laurent Amiotte-Suchet, «Le pasteur pentecôtiste. Un fil conducteur entre le ciel et la terre», se rapproche peut-être le plus de la lecture dominante du charisme dans les sciences sociales. À partir d'un terrain ethnographique effectué dans deux assemblées pentecôtistes de l'Est de la France, il combine l'analyse des histoires de vie des pasteurs à celle du déroulement de réunions d'évangélisation pour construire l'idéaltype du pasteur pentecôtiste, médiateur entre la communauté chrétienne et le créateur. Il illustre ainsi le «travail biographique » du pasteur et son rôle central dans une "économie collective de maîtrise et de gestion de la circulation du Saint Esprit» (p. 107), dont l'authenticité du charisme est tenue pour acquise. Le pasteur devient ainsi un modèle, une inspiration et un guide, "choisi par Dieu lui-même pour permettre à la puissance de l'Esprit de se répandre sur l'assemblée des chrétiens réunis» (p. 112). Cette médiation s'articule en tension avec l'univers protestant évangélique qui privilégie la relation personnelle et intime entre Dieu et l'individu.

\subsection{Le charisme sous le regard de la théologie}

Si le texte de Laurent Amiotte-Suchet évoque à la fois les charismes des membres des assemblées pentecôtistes et celui de leur pasteur, c'est principalement le second qui retient l'attention du sociologue. Les textes des théologiens André Charron et Alain Gignac abordent quant à eux le charisme dans une perspective communautaire, mais dans des contextes et avec des visées différentes.

Dans son article sur «La reconnaissance du charisme d'une communauté religieuse. Le cas de la Congrégation de Sainte-Croix ", André Charron illustre par l'étude d'un cas empirique comment le discernement d'un charisme collectif peut devenir un enjeu institutionnel pour un groupe. Dans une première partie, l'auteur fait appel à Weber, mais il y puise moins un procédé idéaltypique que des éléments de définitions servant à clarifier - 
nous dit l'auteur - le charisme de Basile Moreau, le fondateur de la communauté qui allie charisme personnel et charisme de fonction. Dans une deuxième partie, André Charron témoigne des efforts de définition du charisme par une commission dont il a lui-même fait partie. Le charisme prend ici une autre signification, puisqu'il s'agit de préciser l'identité spécifique ou la personnalité collective d'un groupe religieux. L'exercice exige un travail de mémoire et d'interprétation qui permet de se situer en continuité avec ses origines tout en se projetant dans le futur. Le charisme est là, il s'agit de déterminer son identité particulière, de le discerner et de le réactualiser. Ce charisme collectif puise autant dans le charisme personnel du fondateur que dans les formes sous lesquelles les membres passés et présents ont concrétisé les éléments nécessaires pour préciser la spiritualité, les axes de la mission et le projet de vie communautaire du groupe, contribuant ainsi à mieux cadrer son identité, à accroître sa pertinence et à promouvoir son renouvellement.

Alain Gignac prend son point de départ chez Weber et sa définition du charisme pour étudier les charismes pauliniens. Reconnaissant que la sociologie de Weber est souvent le prisme par lequel les lecteurs de Paul entrevoient le charisme, il s'interroge à savoir si cette approche constitue une piste féconde, par exemple lorsqu'on tente d'éclairer de cette façon la tension présumée entre charisme et institution dans le christianisme primitif: ce serait un cas, parmi d'autres, d'une "problématique wébérienne dans laquelle on a parfois voulu faire entrer de force les éléments du discours paulinien» (p. 156). Avant d'exposer son propre point de vue, Alain Gignac balise le champ sémantique du terme dans les écrits de Paul. Dans le contexte des communautés auxquelles s'adresse l'apôtre, le charisme n'est pas un terme spécialisé pour exprimer la qualité extraordinaire d'une personne en autorité, mais plutôt un mot utilisé de manière originale par Paul pour signifier d'abord le "don de la grâce» et, plus tard, les dons reçus par chacun en vue du bien de la communauté. Cette conception pourrait davantage correspondre, suggère Gignac, à la notion, à peine esquissée, mais présente chez Weber, de "charisme spécifique». Selon certains auteurs évoqués par Gignac, l'idéaltype du chef charismatique se retrouverait cependant, en partie du moins, dans le personnage de Paul ou chez d'autres figures apostoliques; mais l'unanimité est loin d'être faite sur cette question et, de toute manière, l'influence de Paul ou d'autres chefs charismatiques aurait été relativisée assez tôt.

En complément de ce dossier, Jean Duhaime propose un examen de trois approches du messianisme de Qumrân, représentatives des tendances de la 
recherche récente. Plusieurs manuscrits trouvés sur les rives de la mer Morte témoignent en effet, de façon souvent fragmentaire, de la diversité des attentes du judaïsme ancien à l'endroit des figures messianiques, de type charismatique dans certains cas, qui apporteront le salut à une communauté disloquée et soumise au joug de puissances étrangères. Si quelques auteurs limitent leur enquête à l'analyse des textes mentionnant explicitement «un messie ", la plupart procèdent plutôt en construisant un idéaltype, celui du messie ou du "protagoniste eschatologique positif », dont les caractéristiques sont étudiées. Ces approches permettent d'établir une typologie du messianisme et d'explorer la corrélation entre les attentes messianiques et les contextes dans lesquels elles se sont élaborées. Mais le caractère fragmentaire des textes, le traitement assez partiel qu'ils font du sujet et l'insuffisance de données explicites sur l'histoire du ou des groupes dont ils sont issus, ne permettent pas de parvenir à une synthèse cohérente du messianisme de Qumrân et d'en tracer l'évolution de manière précise, confrontant l'analyste aux limites de son objet et de ses instruments méthodologiques.

\section{L'appropriation continue d'un objet-limite}

Ce dossier peut être lu comme une illustration en même temps qu'une mise à l'épreuve de la lecture du charisme qui domine les sciences sociales et humaines depuis quelques décennies et dont l'idéaltype a été esquissé ici. Tout en indiquant les mérites d'une telle approche par le biais d'études empiriques, les auteurs du dossier en déplacent aussi les accents, la critiquent et proposent de nouvelles façons de penser le charisme. S'ils témoignent d'interprétations divergentes, sinon rivales de la notion de charisme qu'ils puisent dans les écrits de Weber, c'est que le charisme occupe une place énigmatique dans les écrits de ce dernier. Ces interprétations renvoient aux questions qui préoccupent, aujourd'hui, les chercheurs. C'est ainsi qu'au delà de la tension entre charisme et institutions, des auteurs ont choisi d'étudier les aiguilleurs du changement (Weichensteller), la singularité d'individus qui ont changé le cours de l'histoire, la proximité entre la croyance traditionnelle et la croyance charismatique, l'identité d'une communauté; plusieurs ont également privilégié une redéfinition de la frontière des sciences sociales et humaines. Les pistes ouvertes par les uns et les autres permettent tantôt d'enrichir la lecture dominante du charisme, tantôt de renouveler la manière de concevoir et d'entrevoir cet objet-limite dans des études empiriques. 
D'un point de vue disciplinaire, le charisme renvoie à divers enjeux. En effet, il a une histoire dont hérite en partie chacun des chercheurs qui le récupère et le reconstruit à sa façon en fonction de sa compréhension personnelle, du domaine où il l'applique et de l'objectif de sa recherche. Le charisme amène la sociologie à s'interroger sur la place de l'individu, de l'exceptionnel, dans l'histoire; il fait aussi écho à la volonté de systématisation souvent associée à la démarche sociologique. Le champ littéraire offre un riche matériau qui invite à réfléchir sur la manière dont le charisme se manifeste et se décrit. La théologie est aussi confrontée à des enjeux analogues qu'elle traite à partir des questions qui lui sont propres, telles que le problème du discernement du charisme de personnes ou de groupes, celui de la réappropriation du concept de charisme dans la discipline après son détournement par la sociologie ou encore son ancrage dans le protestantisme où il s'est déployé sur l'axe de la tension entre le message et l'institution. En ce sens, les textes de ce dossier participent eux aussi au développement continu de la carrière du concept de charisme.

\section{Références}

Albrow, M. (1990), Max Weber's Construction of Social Theory, Basingstoke, Macmillan.

Breuer, S. (1993), "Das Charisma der Vernunft », dans W. GebHard, A. Zingerle et R. N. Ebertz, dir., Charisma. Theorie, Religion, Politik, Berlin/New York, Gruyter, p. 159-184.

Elias, N. (1991), Mozart, sociologie d'un génie, Paris, Seuil.

GeBHARD, W. (1993), «Einleitung. Grundlinien der Entwicklung des Charismakonzeptes in den Sozialwissenschaften ", dans W. GebHard., A. Zingerle, M. N. Ebertz, dir., Charisma. Theorie, Religion, Politik, Berlin/New York, Gruyter, p. 1-12.

HiLDESHeIMER, W. (1991 [1979]), Mozart, Francfort, Suhrkamp.

Rотн, G. (1987), Politische Herrschaft und persönliche Freiheit. Heidelberger Max Weber-Vorlesungen 1983, Francfort, Suhrkamp.

SCHLuChteR, W. (1991), «Umbildung des Charismas. Überlegungen zur Herrschaftssoziologie ", dans Religion und Lebensführung, t. 2, Francfort, Suhrkamp, p. 535-554.

WeIss, J. (2001), «Über interkulturelle Vermittler», dans C. BoHN et H. WILHEMS, dir., Sinngeneratoren: Fremd-und Selbstthematisierung in soziologisch-historischer Perspektive, Konstanz, UVK Verlagsgesellschaft, p. 79-88. 